\title{
Introduction to Loop Quantum Gravity
}

\author{
Abhay Ashtekar*⿰ \\ Institute for Gravitational Physics and Geometry, \\ Physics Department, 104 Davey, Penn State, University Park, PA 16802, USA \\ E-mail: ashtekar@gravity.psu.edu
}

This article is based on the opening lecture at the third quantum geometry and quantum gravity school sponsored by the European Science Foundation and held at Zakopane, Poland in March 2011. The goal of the lecture was to present a broad perspective on loop quantum gravity for young researchers. The first part is addressed to beginning students and the second to young researchers who are already working in quantum gravity.

3rd Quantum Gravity and Quantum Geometry School,

February 28 - March 13, 2011

Zakopane, Poland

*Speaker.

$\dagger$ 


\section{Introduction}

This section, addressed to beginning researchers, is divided into two parts. The first provides a broad historical perspective and the second illustrates key physical and conceptual problems of quantum gravity. Researchers who are already quite familiar with quantum gravity can/should go directly to section 2 ; there will be no loss of continuity.

\subsection{Development of Quantum Gravity: A Bird's Eye View}

The necessity of a quantum theory of gravity was pointed out by Einstein already in a 1916 paper in the Preussische Akademie Sitzungsberichte. He wrote:

- Nevertheless, due to the inneratomic movement of electrons, atoms would have to radiate not only electromagnetic but also gravitational energy, if only in tiny amounts. As this is hardly true in Nature, it appears that quantum theory would have to modify not only Maxwellian electrodynamics but also the new theory of gravitation.

Papers on the subject began to appear in the 1930s most notably by Bronstein, Rosenfeld and Pauli. However, detailed work began only in the sixties. The general developments since then loosely represent four stages, each spanning roughly a decade and a half. In this section, I will present a sketch these developments.

First, there was the beginning: exploration. The goal was to do unto gravity as one would do unto any other physical field [9]. ${ }^{1}$ The electromagnetic field had been successfully quantized using two approaches: canonical and covariant. In the canonical approach, electric and magnetic fields obeying Heisenberg's uncertainty principle are at the forefront, and quantum states naturally arise as gauge-invariant functionals of the vector potential on a spatial three-slice. In the covariant approach on the on the other hand, one first isolates and then quantizes the two radiative modes of the Maxwell field in space-time, without carrying out a (3+1)-decomposition, and the quantum states naturally arise as elements of the Fock space of photons. Attempts were made to extend these techniques to general relativity. In the electromagnetic case the two methods are completely equivalent. Only the emphasis changes in going from one to another. In the gravitational case, however, the difference is profound. This is not accidental. The reason is deeply rooted in one of the essential features of general relativity, namely the dual role of the space-time metric.

To appreciate this point, let us begin with field theories in Minkowski space-time, say Maxwell's theory to be specific. Here, the basic dynamical field is represented by a tensor field $F_{\mu \nu}$ on Minkowski space. The space-time geometry provides the kinematical arena on which the field propagates. The background, Minkowskian metric provides light cones and the notion of causality. We can foliate this space-time by a one-parameter family of space-like three-planes, and analyze how the values of electric and magnetic fields on one of these surfaces determine those on any other surface. The isometries of the Minkowski metric let us construct physical quantities such as fluxes of energy, momentum, and angular momentum carried by electromagnetic waves.

\footnotetext{
${ }^{1}$ Since this introduction is addressed to non-experts, I will generally refer to books and review articles which summarize the state of the art at various stages of development of quantum gravity. References to original papers can be found in these reviews.
} 
In general relativity, by contrast, there is no background geometry. The space-time metric itself is the fundamental dynamical variable. On the one hand it is analogous to the Minkowski metric in Maxwell's theory; it determines space-time geometry, provides light cones, defines causality, and dictates the propagation of all physical fields (including itself). On the other hand it is the analog of the Newtonian gravitational potential and therefore the basic dynamical entity of the theory, similar in this respect to the $F_{\mu \nu}$ of the Maxwell theory. This dual role of the metric is in effect a precise statement of the equivalence principle that is at the heart of general relativity. It is this feature that is largely responsible for the powerful conceptual economy of general relativity, its elegance and its aesthetic beauty, its strangeness in proportion. However, this feature also brings with it a host of problems. We see already in the classical theory several manifestations of these difficulties. It is because there is no background geometry, for example, that it is so difficult to analyze singularities of the theory and to define the energy and momentum carried by gravitational waves. Since there is no a priori space-time, to introduce notions as basic as causality, time, and evolution, one must first solve the dynamical equations and construct a space-time. As an extreme example, consider black holes, whose traditional definition requires the knowledge of the causal structure of the entire space-time. To find if the given initial conditions lead to the formation of a black hole, one must first obtain their maximal evolution and, using the causal structure determined by that solution, ask if the causal past $J^{-}\left(\mathscr{I}^{+}\right)$of its future infinity $\mathscr{I}^{+}$is the entire space-time. If not, space-time contains a black hole and the future boundary of $J^{\left(\mathscr{I}^{+}\right)}$within that space-time is its event horizon. Thus, because there is no longer a clean separation between the kinematical arena and dynamics, in the classical theory substantial care and effort is needed even in the formulation of basic physical questions.

In quantum theory the problems become significantly more serious. To see this, recall first that, because of the uncertainty principle, already in non-relativistic quantum mechanics, particles do not have well-defined trajectories; time-evolution only produces a probability amplitude, $\Psi(x, t)$, rather than a specific trajectory, $x(t)$. Similarly, in quantum gravity, even after evolving an initial state, one would not be left with a specific space-time. In the absence of a space-time geometry, how is one to introduce even habitual physical notions such as causality, time, scattering states, and black holes?

The canonical and the covariant approaches adopted dramatically different attitudes to face these problems. In the canonical approach, one notices that, in spite of the conceptual difficulties mentioned above, the Hamiltonian formulation of general relativity is well-defined and attempts to use it as a stepping stone to quantization. The fundamental canonical commutation relations are to lead us to the basic uncertainty principle. The motion generated by the Hamiltonian is to be thought of as time evolution. The fact that certain operators on the fixed ('spatial') threemanifold commute is supposed to capture the appropriate notion of causality. The emphasis is on preserving the geometrical character of general relativity, on retaining the compelling fusion of gravity and geometry that Einstein created. In the first stage of the program, completed in the early 1960s, the Hamiltonian formulation of the classical theory was worked out in detail by Dirac, Bergmann, Arnowitt, Deser and Misner and others [2, 5, 13, 6, 16]. The basic canonical variable was the 3-metric on a spatial slice. The ten Einstein's equations naturally decompose into two sets: four constraints on the metric and its conjugate momentum (analogous to the equation $\operatorname{Div} \vec{E}=0$ of electrodynamics) and six evolution equations. Thus, in the Hamiltonian formulation, general 
relativity could be interpreted as the dynamical theory of 3-geometries. Wheeler therefore baptized it geometrodynamics $[3,4]$.

In the second stage, this framework was used as a point of departure for quantum theory by Bergmann, Komar, Wheeler DeWitt and others. The basic equations of the quantum theory were written down and several important questions were addressed $[4,16]$. Wheeler also launched an ambitious program in which the internal quantum numbers of elementary particles were to arise from non-trivial, microscopic topological configurations and particle physics was to be recast as 'chemistry of geometry'. However, most of the work in quantum geometrodynamics continued to remain formal; indeed, even today the field theoretic difficulties associated with the presence of an infinite number of degrees of freedom in the Wheeler DeWitt equation remain unresolved. Furthermore, even at the formal level, is has been difficult to solve the quantum Einstein's equations. Therefore, after an initial burst of activity, the quantum geometrodynamics program became stagnant. Interesting results have been obtained by Misner, Wheeler, DeWitt and others in the limited context of quantum cosmology where one freezes all but a finite number of degrees of freedom. However, even in this special case, the initial singularity could not be resolved without additional 'external' inputs into the theory, such as the use of matter violating energy conditions. Sociologically, the program faced another limitation: concepts and techniques which had been so successful in quantum electrodynamics appeared to play no role here. In particular, in quantum geometrodynamics, it is hard to see how gravitons are to emerge, how scattering matrices are to be computed, how Feynman diagrams are to dictate dynamics and virtual processes are to give radiative corrections. To use a well-known phrase [7], the emphasis on geometry in the canonical program "drove a wedge between general relativity and the theory of elementary particles."

In the covariant ${ }^{2}$ approach $[6,8,10]$ the emphasis is just the opposite. Field-theoretic techniques are put at the forefront. The first step in this program is to split the space-time metric $g_{\mu \nu}$ in two parts, $g_{\mu \nu}=\eta_{\mu \nu}+\sqrt{G} h_{\mu \nu}$, where $\eta_{\mu \nu}$ is to be a background, kinematical metric, often chosen to be flat, $G$ is Newton's constant, and $h_{\mu v}$, the deviation of the physical metric from the chosen background, the dynamical field. The two roles of the metric tensor are now split. The overall attitude is that this sacrifice of the fusion of gravity and geometry is a moderate price to pay for ushering-in the powerful machinery of perturbative quantum field theory. Indeed, with this splitting most of the conceptual problems discussed above seem to melt away. Thus, in the transition to the quantum theory it is only $h_{\mu \nu}$ that is quantized. Quanta of this field propagate on the classical background space-time with metric $\eta_{\mu \nu}$. If the background is in fact chosen to be flat, one can use the Casimir operators of the Poincaré group and show that the quanta have spin two and rest mass zero. These are the gravitons. The Einstein-Hilbert Lagrangian tells us how they interact with one another. Thus, in this program, quantum general relativity was first reduced to a quantum field theory in Minkowski space. One could apply to it all the machinery of perturbation theory that had been so successful in particle physics. One now had a definite program to compute amplitudes for various scattering processes. Unruly gravity appeared to be tamed and forced to fit into the mold created to describe other forces of Nature. Thus, the covariant quantization program was more in tune with the mainstream developments in physics at the time. In 1963 Feynman extended

\footnotetext{
${ }^{2}$ In the context of quantum gravity, the term 'covariant' is somewhat misleading because the introduction of a background metric violates diffeomorphism covariance. It is used mainly to emphasize that this approach does not involve a $3+1$ decomposition of space-time.
} 
perturbative methods from quantum electrodynamics to gravity. A few years later DeWitt carried this analysis to completion by systematically formulating the Feynman rules for calculating scattering amplitudes among gravitons and between gravitons and matter quanta. He showed that the theory is unitary order by order in the perturbative expansion. By the early seventies, the covariant approach had led to several concrete results [8].

Consequently, the second stage of the covariant program began with great enthusiasm and hope. The motto was: Go forth, perturb, and expand. The enthusiasm was first generated by the discovery that Yang-Mills theory coupled to fermions is renormalizable (if the masses of gauge particles are generated by a spontaneous symmetry-breaking mechanism). ${ }^{3}$ This led to a successful theory of electroweak interactions. Particle physics witnessed a renaissance of quantum field theory. The enthusiasm spilled over to gravity. Courageous calculations were performed to estimate radiative corrections. Unfortunately, however, this research soon ran into its first road block. The theory was shown to be non-renormalizable when two loop effects are taken into account for pure gravity and already at one loop for gravity coupled with matter [17]. To appreciate the significance of this result, let us return to the quantum theory of photons and electrons. This theory is perturbatively renormalizable. This means that, although individual terms in the perturbation expansion of a physical amplitude may diverge due to radiative corrections involving closed loops of virtual particles, these infinities are of a specific type; they can be systematically absorbed in the values of free parameters of the theory, the fine structure constant and the electron mass. Thus, by renormalizing these parameters, individual terms in the perturbation series can be systematically rendered finite. In quantum general relativity, such a systematic procedure is not available; infinities that arise due to radiative corrections are genuinely troublesome. Put differently, quantum theory acquires an infinite number of undetermined parameters. Although one can still use it as an effective theory in the low energy regime, regarded as a fundamental theory, it has no predictive power at all!

Buoyed, however, by the success of perturbative methods in electroweak interactions, the particle physics community was reluctant to give them up in the gravitational case. In the case of weak interactions, it was known for some time that the observed low energy phenomena could be explained using Fermi's simple four-point interaction. The problem was that this Fermi model led to a non-renormalizable theory. The correct, renormalizable model of Glashow, Weinberg and Salam agrees with Fermi's at low energies but marshals new processes at high energies which improve the ultraviolet behavior of the theory. It was therefore natural to hope that the situation would be similar in quantum gravity. General relativity, in this analogy, would be similar to Fermi's model. The fact that it is not renormalizable was taken to mean that it ignores important processes at high energies which are, however, unimportant at low energies, i.e., at large distances. Thus, the idea was that the correct theory of gravity would differ from general relativity but only at high energies, i.e., near the Planck regime. With this aim, higher derivative terms were added to the EinsteinHilbert Lagrangian. If the relative coupling constants are chosen judiciously, the resulting theory does in fact have a better ultraviolet behavior. Stelle, Tomboulis and others showed that the theory is not only renormalizable but asymptotically free; it resembles the free theory in the high energy

\footnotetext{
${ }^{3}$ In fact DeWitt's quantum gravity work [8] played a seminal role in the initial stages of the extension of perturbative techniques from Abelian to non-Abelian gauge theories.
} 
limit. Thus, the initial hope of 'curing' quantum general relativity was in fact realized. However, it turned out that the Hamiltonian of this theory is unbounded from below, and consequently the theory is drastically unstable! In particular, it violates unitarity; probability fails to be conserved. The success of the electroweak theory suggested a second line of attack. In the approaches discussed above, gravity was considered in isolation. The successful unification of electromagnetic and weak interactions suggested the possibility that a consistent theory would result only when gravity is coupled with suitably chosen matter. The most striking implementation of this viewpoint occurred in supergravity. Here, the hope was that the bosonic infinities of the gravitational field would be canceled by those of suitably chosen fermionic sources, giving us a renormalizable quantum theory of gravity. Much effort went into the analysis of the possibility that the most sophisticated of these theories $-N=8$ supergravity — can be employed as a genuine grand unified theory. ${ }^{4}$ It turned out that cancelations of infinities do occur. Over the last five years or so, there has been a resurgence of interest in this area [55]. It has now been shown that supergravity is finite to four loops even though it contains matter fields coupled to gravity. Furthermore, its Hamiltonian is manifestly positive and the theory is unitary. However, there are several arguments suggesting that the theory would not be renormalizable at seven loops. This is still an open issue but could be settled in the next 2-3 years.

By and large, the canonical approach was pursued by relativists and the covariant approach by particle physicists. In the mid 1980s, both approaches received unexpected boosts. These launched the third phase in the development of quantum gravity.

A group of particle physicists had been studying string theory to analyze strong interactions from a novel angle. The idea was to replace point particles by 1-dimensional extended objects strings - and associate particle-like states with various modes of excitations of the string. Initially there was an embarrassment: in addition to the spin-1 modes characteristic of gauge theories, string theory included also a spin-2, massless excitation. But it was soon realized that this was a blessing in disguise: the theory automatically incorporated a graviton. In this sense, gravity was already built into the theory! However, it was known that the theory had a potential quantum anomaly which threatened to make it inconsistent. In the mid 1980s, Greene and Schwarz showed that there is an anomaly cancelation and perturbative string theory could be consistent in certain spacetime dimensions -26 for a purely bosonic string and 10 for a superstring [20,31]. Since strings were assumed to live in a flat background space-time, one could apply perturbative techniques. However, in this reincarnation, the covariant approach underwent a dramatic revision. Since it is a theory of extended objects rather than point particles, the quantum theory has brand new elements; it is no longer a local quantum field theory. The field theoretic Feynman diagrams are replaced by world-sheet diagrams. This replacement dramatically improves the ultraviolet behavior and, although explicit calculations have been carried out only at 2 or 3 loop order, in the string theory community it is widely believed that the perturbation theory is finite to all orders; it does not even have to be renormalized. The theory is also unitary. It has a single, new fundamental constant

\footnotetext{
${ }^{4}$ For a number of years, there was a great deal of confidence, especially among particle physicists, that supergravity was on the threshold of providing the complete quantum gravity theory. For instance, in the centennial celebration of Einstein's birthday at the Institute of Advanced Study, Princeton [14] — the proceedings of which were videotaped and archived for future historians and physicists - there were two talks on quantum gravity, both devoted to supergravity. A year later, in his Lucasian Chair inaugural address Hawking [15] suggested that end of theoretical physics was in sight because $N=8$ supergravity was likely to be the final theory.
} 
- the string tension - and, since various excited modes of the string represent different particles, there is a built-in principle for unification of all interactions! $!^{5}$ From the viewpoint of local quantum field theories that particle physicists have used in studying electroweak and strong interactions, this mathematical structure seems almost magical. Therefore there has been a long-standing hope in the string community that this theory would encompass all of fundamental physics; it would be the 'theory of everything'.

Unfortunately, it soon became clear that string perturbation theory also faces some serious limitations. Perturbative finiteness would imply that each term in the perturbation series is ultra-violet finite. ${ }^{6}$ However Gross and Periwal have shown that in the case of bosonic strings, when summed, the series diverges and does so uncontrollably. (Technically, it is not even Borel-summable.) They also gave arguments that the conclusion would not be changed if one uses superstrings instead. Independent support for these arguments has come from work on random surfaces due to Ambjorn and others. One might wonder why the divergence of the sum should be regarded as a serious failure of the theory. After all, in quantum electrodynamics, the series is also believed to diverge. Recall however that quantum electrodynamics is an inherently incomplete theory. It ignores many processes that come into play at high energies or short distances. In particular, it completely ignores the microstructure of space-time and simply assumes that space-time can be approximated by a smooth continuum even below the Planck scale. Therefore, it can plead incompleteness and shift the burden of this infinity to a more complete theory. A 'theory of everything' on the other hand, has nowhere to hide. It cannot plead incompleteness and shift its burden. It must face the Planck regime squarely. If the theory is to be consistent, it must have key non-perturbative structures.

The current and the fourth stage of the particle physics motivated approaches to quantum gravity is largely devoted to unraveling such structures and using them to make unsuspected connections between gravity and other areas of physics such as fluid dynamics and high temperature superconductivity. It is widely believed that the AdS/CFT conjecture provides a non-perturbative definition of string theory on space-times satisfying certain boundary conditions [49]. More precisely, in this correspondence string theory on asymptotically AdS bulk space-times is taken to be equivalent to certain gauge theories on its boundary. However, from the perspective of quantum gravity, this approach has some serious limitations. First, a negative cosmological constant in the bulk is essential to this correspondence while the observed cosmological constant is positive. Considerable effort were made initially to extend the ideas to a positive or zero cosmological constant but they have not had notable success. Second, the bulk space-time is 10 dimensional. One can compactify the unwanted dimensions using n-spheres, but the compactified directions cannot be microscopic; the correspondence requires that the radius of these spheres should equal the cosmological radius! So if one just looks around, one should see these large macroscopic dimensions. Consequently, the non-perturbative string theory defined through the conjecture has little to do with the macroscopic world we live in. Finally, even if one overlooks this issue and considers space-times with higher macroscopic dimensions, the AdS/CFT duality is yet to shed light on the conceptually central is-

\footnotetext{
${ }^{5}$ To date, none of the low energy reductions appears to correspond to the world we actually observe. Nonetheless, string theory has provided us with a glimpse of an entirely new vista: the concrete possibility that unification could be brought about by a tightly woven, non-local theory.

${ }^{6} \mathrm{But}$ it does appear that there are infrared divergences. As in QED, these are regarded as 'harmless' for calculation of physical effects. I thank Ashoke Sen for discussions on this issue.
} 
sues such as the space-time structure inside the horizon of an evaporating black hole or the fate of big-bang type cosmological singularities. The recent thrust — and the strength - of these developments is, rather, that they enable one to use known techniques from gravity and supergravity to solve some of the difficult mathematical problems encountered in the strong coupling regimes of field theories describing non-gravitational systems.

On the relativity side, the third stage began with the following observation: the geometrodynamics program laid out by Dirac, Bergmann, Wheeler and others simplifies significantly if we regard a spatial connection - rather than the 3-metric - as the basic object. In fact we now know that, among others, Einstein and Schrödinger had recast general relativity as a theory of connections already in the fifties.(For a brief account of this fascinating history, see [33].) However, they used the 'Levi-Civita connection' that features in the parallel transport of vectors and found that the theory becomes rather complicated. This episode had been forgotten and connections were re-introduced afresh in the mid 1980s [22]. ${ }^{7}$ However, now these are 'spin-connections' required to parallel propagate spinors, and they turn out to simplify Einstein's equations considerably. For example, the dynamical evolution dictated by Einstein's equations can now be visualized simply as a geodesic motion on the space of spin-connections (with respect to a natural metric extracted from the constraint equations). Since general relativity is now regarded as a dynamical theory of connections, this reincarnation of the canonical approach is called 'connection-dynamics'.

Perhaps the most important advantage of the passage from metrics to connections is that the phase-space of general relativity is now the same as that of gauge theories [22, 26]. The 'wedge between general relativity and the theory of elementary particles' that Weinberg referred to largely disappears without having to sacrifice the geometrical essence of general relativity. One could now import into general relativity techniques that have been highly successful in the quantization of gauge theories. At the kinematic level, then, there is a unified framework to describe all four fundamental interactions. The dynamics, of course, depends on the interaction. In particular, while there is a background space-time geometry in electroweak and strong interactions, there is none in general relativity. Therefore, qualitatively new features arise. These were exploited in the late eighties and early nineties to solve simpler models - general relativity in $2+1$ dimensions [22, 23, 29]; linearized gravity clothed as a gauge theory [22]; and certain cosmological models. To explore the physical, 3+1 dimensional theory, a 'loop representation' was introduced by Rovelli and Smolin. Here, quantum states were taken to be suitable functions of loops on the 3-manifold. ${ }^{8}$ This led to a number of interesting and intriguing results, particularly by Gambini, Pullin and

\footnotetext{
${ }^{7}$ This reformulation used (anti-)self-dual connections which are complex. These have a direct interpretation in terms space-time geometry and also render the constraint equations polynomial in the basic variables. This simplicity was regarded as crucial for passage to quantum theory. However, one is then faced with the task of imposing appropriate quantum 'reality conditions' to ensure that the classical limit is real general relativity. Barbero introduced real connection variables by replacing the $\pm i$ in the expression of the (anti-)self-dual connections with a real parameter $\beta$. However, now the connection does not have a natural space-time interpretation and the constraints are no longer polynomial in the basic variables. But the strategy became viable after Thiemann introduced novel ideas to handle quantization of the specific non-polynomial terms that now feature in the constraints. Since then this strategy has become crucial because the rigorous functional calculus on the space of connections has so far been developed only for real connections. Immirzi suggested that the value of $\beta$ could be chosen so that the leading term in black hole entropy is precisely (area/ $4 \ell_{\mathrm{Pl}}^{2}$ ). That is why $\beta$ (which is often denoted by $\gamma$ in later papers) is referred to as the Barbero-Immirzi parameter.

${ }^{8}$ This is the origin of the name 'loop quantum gravity'. The loop representation played an important role in the initial stages. Although this is no longer the case in the current, fourth phase, the name is still used to distinguish this
} 
their collaborators, relating knot theory and quantum gravity [28]. Thus, there was rapid and unanticipated progress in a number of directions which rejuvenated the canonical quantization program. Since the canonical approach does not require the introduction of a background geometry or use of perturbation theory, and because one now has access to fresh, non-perturbative techniques from gauge theories, in relativity circles there is a hope that this approach may lead to well-defined, non-perturbative quantum general relativity, or its supersymmetric version, supergravity.

However, a number of these considerations remained rather formal until mid-nineties. Passage to the loop representation required an integration over the infinite dimensional space of connections and the formal methods were insensitive to possible infinities lurking in the procedure. Indeed, such integrals are notoriously difficult to perform in interacting field theories. To pay due respect to the general covariance of Einstein's theory, one needed diffeomorphism invariant measures and there were folk-theorems to the effect that such measures did not exist!

Fortunately, the folk-theorems turned out to be incorrect. To construct a well-defined theory capable of handling field theoretic issues, a quantum theory of Riemannian geometry was systematically constructed in the mid-nineties [38]. This launched the fourth (and the current) stage in the canonical approach. Just as differential geometry provides the basic mathematical framework to formulate modern gravitational theories in the classical domain, quantum geometry provides the necessary concepts and techniques in the quantum domain. It is a rigorous mathematical theory which enables one to perform integration on the space of connections for constructing Hilbert spaces of states and to define geometric operators corresponding, e.g. to areas of surfaces and volumes of regions, even though the classical expressions of these quantities involve non-polynomial functions of the Riemannian metric. There are no infinities. One finds that, at the Planck scale, geometry has a definite discrete structure. Its fundamental excitations are 1-dimensional, rather like polymers, and the space-time continuum arises only as a coarse-grained approximation. The fact that the structure of space-time at Planck scale is qualitatively different from Minkowski background used in perturbative treatments reinforced the idea that quantum general relativity (or supergravity) may well be non-perturbatively finite. Quantum geometry effects have already been shown to resolve the big-bang singularity and solve some of the long-standing problems associated with black holes. (See lectures by Giesel, Sahlmann and Singh at this School.)

Over the last six years, another frontier has advanced in loop quantum gravity: spin foams (and the associated development of group field theory) which provide a sum over histories formulation $[35,39,48]$. The new element here is that the histories that enter the sum are quantum geometries of a specific type; they can be regarded as the 'time evolution' of the polymer-like quantum 3 geometries that emerged in the canonical approach. So far the sum has not been systematically derived starting from the classical theory as one generally does in, say, gauge theories. Rather, one uses semi-heuristic considerations to arrive at a definition of the 'transition amplitudes' and then explores physical properties of the resulting quantum theory. There are detailed arguments to the effect that one recovers the Einstein Hilbert action in an appropriate limit. Furthermore, although the underlying theory is diffeomorphism invariant, given a suitable 'boundary state', there is a conceptual framework to calculate n-point functions normally used in perturbative treatments. Information about the background space-time on which these n-point functions live is encoded in

approach from others. 
the chosen 'boundary state'. However, a number of important problems still remain. The status is described in detail in the lectures by Rovelli, Speziale, Baratin, Perini, Fairbairn, Bianchi and Kaminski at this school.

The first three stages of developments in quantum gravity taught us many valuable lessons. Perhaps the most important among them is the realization that perturbative, field theoretic methods which have been so successful in other branches of physics are inadequate to construct quantum gravity. The assumption that space-time can be replaced by a smooth continuum at arbitrarily small scales leads to inconsistencies. We can neither ignore the microstructure of space-time nor presuppose its nature. We must let quantum gravity itself reveal this structure to us.

For brevity and to preserve the flow of discussion, I have restricted myself to the 'main-stream' programs whose development can be continuously tracked over several decades. However, I would like to emphasize that there are a number of other highly original approaches - particularly, the Euclidean path integral approach [12], Regge calculus [24], asymptotic safety scenarios [47], discrete approaches [50], causal dynamical triangulations [30, 40], twistor theory $[11,21,43]$ and the theory of $\mathrm{H}$-spaces [18], asymptotic quantization [19], non-commutative geometry [25], causal sets $[37,44]$ and Topos theory $[51,56]$. Ideas underlying several of these approaches are inter-related and some of them lie at the foundation of other avenues. This is particularly true of the path integral approach pioneered by Misner, and developed in much greater detail in the Euclidean context by Hawking, Hartle, Halliwell and others. Ideas developed in this approach have provided the point of departure for the ongoing developments in causal dynamical triangulations, asymptotic safety and spin foams.

\subsection{Physical Questions of Quantum Gravity}

Approaches to quantum gravity face two types of issues: Problems that are 'internal' to individual programs and physical and conceptual questions that underlie the whole subject. Examples of the former are: Incorporation of physical — rather than half flat- gravitational fields in the twistor program, mechanisms for breaking of supersymmetry and dimensional reduction in string theory, and issues of space-time covariance in the canonical approach. In this sub-section, I will focus on the second type of issues by recalling some of the long standing issues that any satisfactory quantum theory of gravity should address.

- The Big-Bang and other singularities: It is widely believed that the prediction of a singularity, such as the big-bang of classical general relativity, is primarily a signal that the physical theory has been pushed beyond the domain of its validity. A key question to any quantum gravity theory, then, is: What replaces the big-bang? Are the classical geometry and the continuum picture only approximations, analogous to the 'mean (magnetization) field' of ferro-magnets? If so, what are the microscopic constituents? What is the space-time analog of a Heisenberg quantum model of a ferro-magnet? When formulated in terms of these fundamental constituents, is the evolution of the quantum state of the universe free of singularities? General relativity predicts that the space-time curvature must grow unboundedly as we approach the big-bang or the big-crunch but we expect the quantum effects, ignored by general relativity, to intervene, making quantum gravity indispensable 
before infinite curvatures are reached. If so, what is the upper bound on curvature? How close to the singularity can we 'trust' classical general relativity? What can we say about the 'initial conditions', i.e., the quantum state of geometry and matter that correctly describes the big-bang? If they have to be imposed externally, is there a physical guiding principle?

- Black holes: In the early seventies, using imaginative thought experiments, Bekenstein argued that black holes must carry an entropy proportional to their area [12, 34, 41]. About the same time, Bardeen, Carter and Hawking (BCH) showed that black holes in equilibrium obey two basic laws, which have the same form as the zeroth and the first laws of thermodynamics, provided one equates the black hole surface gravity $\kappa$ to some multiple of the temperature $T$ in thermodynamics and the horizon area $a_{\text {hor }}$ to a corresponding multiple of the entropy $S[12,34,41]$. However, at first this similarity was thought to be only a formal analogy because the $\mathrm{BCH}$ analysis was based on classical general relativity and simple dimensional considerations show that the proportionality factors must involve Planck's constant $\hbar$. Two years later, using quantum field theory on a black hole background space-time, Hawking showed that black holes in fact radiate quantum mechanically as though they are black bodies at temperature $T=\hbar \kappa / 2 \pi[12,27]$. Using the analogy with the first law, one can then conclude that the black hole entropy should be given by $S_{\mathrm{BH}}=a_{\mathrm{hor}} / 4 G \hbar$. This conclusion is striking and deep because it brings together the three pillars of fundamental physics - general relativity, quantum theory and statistical mechanics. However, the argument itself is a rather hodge-podge mixture of classical and semi-classical ideas, reminiscent of the Bohr theory of atom. A natural question then is: what is the analog of the more fundamental, Pauli-Schrödinger theory of the Hydrogen atom? More precisely, what is the statistical mechanical origin of black hole entropy? What is the nature of a quantum black hole and what is the interplay between the quantum degrees of freedom responsible for entropy and the exterior curved geometry? Can one derive the Hawking effect from first principles of quantum gravity? Is there an imprint of the classical singularity on the final quantum description, e.g., through 'information loss'?

- Planck scale physics and the low energy world: In general relativity, there is no background metric, no inert stage on which dynamics unfolds. Geometry itself is dynamical. Therefore, as indicated above, one expects that a fully satisfactory quantum gravity theory would also be free of a background space-time geometry. However, of necessity, a background independent description must use physical concepts and mathematical tools that are quite different from those of the familiar, low energy physics. A major challenge then is to show that this low energy description does arise from the pristine, Planckian world in an appropriate sense, bridging the vast gap of some 16 orders of magnitude in the energy scale. In this 'top-down' approach, does the fundamental theory admit a 'sufficient number' of semi-classical states? Do these semi-classical sectors provide enough of a background geometry to anchor low energy physics? Can one recover the familiar description? If the answers to these questions are in the affirmative, can one pin point why the standard 'bottom-up' perturbative approach fails? That is, what is the essential feature which makes the fundamental description mathematically coherent but is absent in the standard perturbative quantum gravity?

There are of course many more challenges: adequacy of standard quantum mechanics, the issue of time, of measurement theory and the associated questions of interpretation of the quantum framework, the issue of diffeomorphism invariant observables and practical methods of computing 
their properties, convenient ways of computing time evolution and S-matrices, exploration of the role of topology and topology change, etc, etc. In loop quantum gravity described in the rest of this chapter, one adopts the view that the three issues discussed in detail are more basic from a physical viewpoint because they are rooted in general conceptual questions that are largely independent of the specific approach being pursued. Indeed they have been with us longer than any of the current leading approaches.

\section{Loop quantum gravity}

In this section, I will summarize the overall viewpoint, achievements, challenges and opportunities underlying loop quantum gravity. The emphasis is on structural and conceptual issues. I would like to emphasize that this is not a comprehensive review. The choice of the material was guided by two principles: i) Choosing illustrative topics to provide glimpses of directions that have been and are being pursued; and ii) Avoiding issues that I do not understand well or those would need detours to explain strengths and weaknesses.

Detailed treatments of the subject can be found in lectures by Giesel, Sahlmann, Rovelli and Singh in these proceedings and even more complete and more technical accounts in [38, 39, 45] and references therein. (The development of the subject can be seen by following older monographs $[22,26,28]$.) For a treatment at a more elementary (i.e. advanced undergraduate) level, see [53].

\subsection{Viewpoint}

In loop quantum gravity, one takes the central lesson of general relativity seriously: gravity is geometry whence, in a fundamental quantum gravity theory, there should be no background metric. Geometry and matter should both be 'born quantum mechanically'. Thus, in contrast to approaches developed by particle physicists, one does not begin with quantum matter on a background geometry and use perturbation theory to incorporate quantum effects of gravity. There is a manifold but no metric, or indeed any other physical fields, in the background. ${ }^{9}$

In classical gravity, Riemannian geometry provides the appropriate mathematical language to formulate the physical, kinematical notions as well as the final dynamical equations. This role is now taken by quantum Riemannian geometry. In the classical domain, general relativity stands out as the best available theory of gravity, some of whose predictions have been tested to an amazing degree of accuracy, surpassing even the legendary tests of quantum electrodynamics. Therefore, it is natural to ask: Does quantum general relativity, coupled to suitable matter (or supergravity, its supersymmetric generalization) exist as consistent theories non-perturbatively? There is no implication that such a theory would be the final, complete description of Nature. Nonetheless, this is a fascinating and important open question in its own right.

As explained in section 1.1, in particle physics circles the answer is often assumed to be in the negative, not because there is concrete evidence against non-perturbative quantum gravity, but because of the analogy to the theory of weak interactions. There, one first had a 4-point interaction

\footnotetext{
${ }^{9}$ In $2+1$ dimensions, although one begins in a completely analogous fashion, in the final picture one can get rid of the background manifold as well. Thus, the fundamental theory can be formulated combinatorially [22, 23]. While some steps have been taken to achieve this in 3+1 dimensions, by considering 'abstract' spin networks in the canonical approach and 2-complexes in spin foams, one still needs a more complete handle on the underlying mathematics.
} 
model due to Fermi which works quite well at low energies but which fails to be renormalizable. Progress occurred not by looking for non-perturbative formulations of the Fermi model but by replacing the model by the Glashow-Salam-Weinberg renormalizable theory of electro-weak interactions, in which the 4-point interaction is replaced by $W^{ \pm}$and $Z$ propagators. Therefore, it is often assumed that perturbative non-renormalizability of quantum general relativity points in a similar direction. However this argument overlooks the crucial fact that, in the case of general relativity, there is a qualitatively new element. Perturbative treatments pre-suppose that the space-time can be assumed to be a continuum at all scales of interest to physics under consideration. This assumption is safe for weak interactions. In the gravitational case, on the other hand, the scale of interest is the Planck length $\ell_{\mathrm{Pl}}$ and there is no physical basis to pre-suppose that the continuum picture should be valid down to that scale. The failure of the standard perturbative treatments may largely be due to this grossly incorrect assumption and a non-perturbative treatment which correctly incorporates the physical micro-structure of geometry may well be free of these inconsistencies.

Are there any situations, outside loop quantum gravity, where such physical expectations are borne out in detail mathematically? The answer is in the affirmative. There exist quantum field theories (such as the Gross-Neveu model in three dimensions) in which the standard perturbation expansion is not renormalizable although the theory is exactly soluble! Failure of the standard perturbation expansion can occur because one insists on perturbing around the trivial, Gaussian point rather than the more physical, non-trivial fixed point of the renormalization group flow. Interestingly, thanks to recent work by Reuter, Lauscher, Percacci, Perini and others there is now non-trivial and growing evidence that situation may be similar in Euclidean quantum gravity. Impressive calculations have shown that pure Einstein theory may also admit a non-trivial fixed point $[47,52]$. Furthermore, the requirement that the fixed point should continue to exist in presence of matter constrains the couplings in non-trivial and interesting ways [42].

However, as indicated in the Introduction, even if quantum general relativity did exist as a mathematically consistent theory, there is no a priori reason to assume that it would be the 'final' theory of all known physics. In particular, as is the case with classical general relativity, while requirements of background independence and general covariance do restrict the form of interactions between gravity and matter fields and among matter fields themselves, the theory would not have a built-in principle which determines these interactions. Put differently, such a theory would not be a satisfactory candidate for unification of all known forces. However, just as general relativity has had powerful implications in spite of this limitation in the classical domain, quantum general relativity should have qualitatively new predictions, pushing further the existing frontiers of physics. Indeed, unification does not appear to be an essential criterion for usefulness of a theory even in other interactions. QCD, for example, is a powerful theory even though it does not unify strong interactions with electro-weak ones. Furthermore, the fact that we do not yet have a viable candidate for the grand unified theory does not make QCD any less useful.

\subsection{Advances}

From the historical and conceptual perspectives of section 1, loop quantum gravity has had several successes. Thanks to the systematic development of quantum geometry, several of the roadblocks encountered by quantum geometrodynamics were removed. Functional analytic issues related to the presence of an infinite number of degrees of freedom are now faced squarely. Inte- 
grals on infinite dimensional spaces are rigorously defined and the required operators have been systematically constructed. Thanks to this high level of mathematical precision, the Hamiltonian and the spin foam programs in loop quantum gravity have leaped past the 'formal' stage of development. More importantly, although key issues related to quantum dynamics still remain, it has been possible to use the parts of the program that are already well established to extract useful and highly non-trivial physical predictions. In particular, some of the long standing issues about the nature of the big-bang, physics of the very early universe, properties of quantum black holes, giving meaning to the n-point functions in a background independent framework have been resolved. In this sub-section, I will further clarify some conceptual issues and discuss some recent advances.

- Quantum geometry. The specific quantum Riemannian geometry underlying loop quantum gravity predicts that eigenvalues of geometric operators — such as areas of 2-surfaces and volumes of 3-dimensional regions - are discrete. Thus, continuum underlying general relativity is only a coarse grained approximation. What is the direct physical significance of this specific discreteness? Recall first that, in the classical theory, differential geometry simply provides us with formulas to compute areas of surfaces and volumes of regions in a Riemannian manifold. To turn these quantities into physical observables of general relativity, one has to define the surfaces and regions operationally, e.g. by focusing on surfaces of black holes or regions in which matter fields are non-zero. Once this is done, one can simply use the formulas supplied by differential geometry to calculate values of these observable. The situation is rather similar in loop quantum gravity. For instance, the area of the isolated horizon is a Dirac observable in the classical theory and the application of the quantum geometry area formula to this surface leads to physical results. In $2+1$ dimensions, Freidel, Noui and Perez have introduced point particles coupled to gravity. The physical distance between these particles is again a Dirac observable. When used in this context, the spectrum of the length operator has direct physical meaning. In all these situations, the operators and their eigenvalues correspond to the 'proper' lengths, areas and volumes of physical objects, measured in the rest frames. Finally sometimes questions are raised about compatibility between discreteness of these eigenvalues and Lorentz invariance. As was emphasized by Rovelli and Speziale, there is no tension whatsoever: it suffices to recall that discreteness of eigenvalues of the angular momentum operator $\hat{J}_{z}$ of non-relativistic quantum mechanics is perfectly compatible with the rotational invariance of that theory.

- Quantum cosmology. In Friedmann-Lemaitre-Robertson-Walker (FLRW) models, loop quantum gravity has resolved the long-standing physical problem of the fate of the big-bang in quantum gravity [54]. Work by Bojowald, Ashtekar, Pawlowski, Singh and others has shown that nonperturbative effects originating in quantum geometry create an effective repulsive force which is negligible when the curvature falls significantly below the Planck scale but rises very quickly and dramatically in the deep Planck regime to overcome the classical gravitational attraction, thereby replacing the big-bang by a quantum bounce. The same is true with the big-crunch singularity in the closed models. More generally, using effective equations, Singh has shown that these quantum geometry effects also resolve all strong curvature singularities in homogeneous isotropic models where matter sources have an equation of state of the type $p=p(\rho)$, including the exotic singularities such as the big-rip. (These can occur with non-standard matter, still described by an equation 
of state $p=p(\rho))$.

A proper treatment of anisotropies (i.e. Bianchi models) has long been a highly non-trivial issue in general bouncing scenarios because the anisotropic shears dominate in Einstein's equations in the contracting phase before the bounce, diverging (as $1 / a^{6}$ which is) faster than, say, the dust or radiation matter density. Therefore, if anisotropies are added even as a perturbation to a FLRW model, they tend to grow unboundedly. What is the situation in loop quantum cosmology? The issue turned out to be quite subtle and there were some oversights at first. But a careful examination by Ashtekar, Wilson-Ewing and others has shown that the singularity is again resolved: any time a shear scalar - a potential for the Weyl curvature - or matter density approaches the Planck regime, the repulsive force of quantum geometry grows to dilute it. As in the isotropic case, effective equations can again be used to gain physical insights. In particular they show that the matter density is again bounded above. Singularity resolution in these Bianchi models is also important from a more general consideration. There is a conjecture due to Belinskii, Khalatnikov and Lifshitz (BKL) that says that as one approaches a space-like singularity in classical general relativity, 'the terms containing time derivatives dominate over those containing spatial derivatives, so that the dynamics of the gravitational field at any one spatial point are better and better approximated by the dynamics of Bianchi models'. By now considerable evidence has accumulated in support of the BKL conjecture and it is widely believed to be essentially correct. One might therefore hope that the singularity resolution in the Bianchi models in loop quantum cosmology has opened a door to showing that all strong curvature, space-like singularities are resolved by the quantum geometry effects underlying loop quantum gravity.

Finally, the simplest type of (non-linear) inhomogeneous models - the 1-polarization Gowdy space-times - have also been analyzed in detail. These models were studied extensively in the early quantum gravity literature, prior to the advent of LQC. In all cases the singularity had persisted. A systematic study in the context of loop quantum cosmology was initiated by Mena, Garay, MartinBenito, Vehlino and others by making an astute use of the fact that the homogeneous modes of the model correspond to a Bianchi I space-time. Once again, the underlying quantum geometry resolves the big-bang singularity.

I will conclude with the discussion of a conceptual point. In general relativity, non-singular, bouncing models can be and have been constructed by using matter fields that violate energy conditions. In loop quantum cosmology, by contrast, matter fields satisfy all energy conditions. How can the theory then evade singularity theorems of Penrose, Hawking and others? It does so because the quantum geometry effects modify the geometric, left hand side of Einstein's equations, whence these theorems are inapplicable. However there are more recent singularity theorems due to Borde, Guth and Vilenkin which do not refer to field equations at all. How are these evaded? These theorems were motivated by inflationary scenario and therefore assume that the universe bas been eternally undergoing an expansion. In loop quantum cosmology, even with an inflationary potential, the pre-bounce branch is contracting. Thus again the singularity is avoided because the solutions violate a key assumption of these theorems as well.

- Quantum Horizons. Loop quantum cosmology illuminates dynamical ramifications of quantum geometry but within the context of mini and midi superspaces where an infinite number of degrees of freedom are frozen. The application to the black hole entropy problem is complemen- 
tary in that one considers the full theory but probes consequences of quantum geometry which are not sensitive to full quantum dynamics. I will discuss this topic in a little more detail because it was not covered in any of the main lectures at this school.

As explained in the Introduction, since mid-seventies, a key question in the subject has been: What is the statistical mechanical origin of the entropy $S_{\mathrm{BH}}=\left(a_{\mathrm{hor}} / 4 \ell_{\mathrm{Pl}}^{2}\right)$ of large black holes? What are the microscopic degrees of freedom that account for this entropy? This relation implies that a solar mass black hole must have exp $10^{77}$ quantum states, a number that is huge even by the standards of statistical mechanics. Where do all these states reside? To answer these questions, in the early 1990s Wheeler had suggested the following heuristic picture, which he christened 'It from Bit'. Divide the black hole horizon into elementary cells, each with one Planck unit of area, $\ell_{\mathrm{Pl}}^{2}$ and assign to each cell two microstates, or one 'Bit'. Then the total number of states $\mathscr{N}$ is given by $\mathscr{N}=2^{n}$ where $n=\left(a_{\mathrm{hor}} / \ell_{\mathrm{Pl}}^{2}\right)$ is the number of elementary cells, whence entropy is given by $S=\ln \mathscr{N} \sim a_{\text {hor }}$. Thus, apart from a numerical coefficient, the entropy ('It') is accounted for by assigning two states ('Bit') to each elementary cell. This qualitative picture is simple and attractive. But can these heuristic ideas be supported by a systematic analysis from first principles?

Ashtekar, Baez, Corichi and Krasnov used quantum geometry to provide such an analysis. The first step was to analyze the structure of 'isolated horizons' in general relativity [41] and use it in conjunction to quantum geometry to define an isolated quantum horizon. To probe its properties, one has to combine the isolated horizon boundary conditions from classical general relativity and quantum Riemannian geometry of loop quantum gravity with the Chern-Simons theory on a punctured sphere, the theory of a non-commutative torus and subtle considerations involving mapping class groups. This detailed analysis showed that, while qualitative features of Wheeler's picture are borne out, geometry of a quantum horizon is much more subtle. First, while Wheeler's ideas hold for any 2 -surface, the loop quantum gravity calculation requires a quantum horizon. Second, basic features of both of Wheeler's arguments undergo a change: i) the elementary cells do not have Planck area; values of their area are dictated by the spectrum, $\sim \sqrt{j(j+1)}$, of the area operator in loop quantum gravity, where $j$ is a half integer; ii) individual cells carry much more than just one 'bit' of information; the number of states associated with any one cell is $2 j+1$.

Nonetheless, a careful counting of states by Lewandowski, Domagala, Meissner and others has shown that the number of microstates is again proportional to the area of the isolated horizon. To get the exact numerical factor of $1 / 4$, one has to fix the Barbero-Immirzi parameter of loop quantum gravity to a specific value. One can use a specific type of isolated horizon for this -e.g. the spherically symmetric one with zero charge, or the cosmological one in the de Sitter space-time. Once the value of the parameter is fixed, one gets the correct numerical coefficient in the leading order contribution for isolated horizons with arbitrary mass and angular momentum moments, charge, etc. (One also obtains a precise logarithmic sub-leading correction, whose coefficient does not depend on the Barbero-Immirzi parameter.) The final result has two significant differences with respect to the string theory calculations: i) one does not require near-extremality; one can handle ordinary 4-dimensional black holes of direct astrophysical interest which may be distorted and/or rotating; and, ii) one can simultaneously incorporate cosmological horizons for which thermodynamics considerations also apply [12].

Why does this value of the Barbero-Immirzi parameter not depend on non-gravitational charges? This important property can be traced back to a key consequence of the isolated horizon boundary 
conditions: detailed calculations show that only the gravitational part of the symplectic structure has a surface term at the horizon; the matter symplectic structures have only volume terms. (Furthermore, the gravitational surface term is insensitive to the value of the cosmological constant.) Consequently, there are no independent surface quantum states associated with matter. This provides a natural explanation of the fact that the Hawking-Bekenstein entropy depends only on the horizon area and is independent of electro-magnetic (or other) charges. (For more detailed accounts of these results, see $[38,41]$.)

Over the last three years there has been a resurgence of interest in the subject, thanks to the impressive use of number theory techniques by Barbero, Villasenor, Agullo, Borja, Diaz-Polo and to sharpen and very significantly extend the counting of horizon states. These techniques have opened new avenues to further explore the microstates of the quantum horizon geometry through contributions by Perez, Engle, Noui, Pranzetti, Ghosh, Mitra, Kaul, Majumdar and others.

To summarize, as in other approaches to black hole entropy, concrete progress could be made in loop quantum gravity because: i) the analysis does not require detailed knowledge of how quantum dynamics is implemented in full theory, and, ii) restriction to large black holes implies that the Hawking radiation is negligible, whence the black hole surface can be modeled by an isolated horizon [41]. The states responsible for entropy have a direct interpretation in space-time terms: they refer to the geometry of the quantum, isolated horizon.

- Quantum Einstein's equations in the canonical framework. The challenge of quantum dynamics in the full theory is to find solutions to the quantum constraint equations and endow these physical states with the structure of an appropriate Hilbert space. The general consensus in the loop quantum gravity community is that while the situation is well-understood for the Gauss and diffeomorphism constraints, it is far from being definitive for the Hamiltonian constraint. Non-trivial development due to Thiemann is that well-defined candidate operators representing the Hamiltonian constraint exist on the space of solutions to the Gauss and diffeomorphism constraints [45]. However there are several ambiguities [38] and, unfortunately, we do not understand the physical meaning of choices made to resolve them. Detailed analysis in the limited context of loop quantum cosmology has shown that choices which appear to be mathematically natural can nonetheless lead to unacceptable physical consequences such as departures from general relativity in completely tame situations with low curvature [54]. Therefore, much more work is needed in the full theory.

The current status can be summarized as follows. Four main avenues have been pursued to construct and solve the quantum Hamiltonian constraint. The first is the 'Master constraint program' introduced by Thiemann [45]. The idea here is to avoid using an infinite number of Hamiltonian constraints $\mathscr{S}(N)=\int N(x) \mathscr{S}(x) d^{3} x$, each smeared by a so-called 'lapse function' $N$. Instead, one squares the integrand $\mathscr{S}(x)$ itself in an appropriate sense and then integrates it on the 3-manifold $M$. In simple examples, this procedure leads to physically viable quantum theories. However, in loop quantum gravity the procedure does not remove any of the ambiguities in the definition of the Hamiltonian constraint. Rather, if the ambiguities are resolved, the principal strength of the strategy lies in its potential to complete the last step in quantum dynamics: finding the physically appropriate scalar product on physical states. The general philosophy is similar to that advocated by John Klauder over the years in his approach to quantum gravity based on coherent states [36]. A second strategy to solve the quantum Hamiltonian constraint is due to Gambini, Pullin and their 
collaborators. It builds on their extensive work on the interplay between quantum gravity and knot theory [28]. The more recent of these developments use the relatively new invariants of intersecting knots discovered by Vassiliev. This is a novel approach which furthermore has a potential of enhancing the relation between topological field theories and quantum gravity. As our knowledge of invariants of intersecting knots deepens, this approach could provide increasingly significant insights. In particular, it has the potential of leading to a formulation of quantum gravity which does not refer even to a background manifold (see footnote 9).

The third approach comes from spin-foam models [35, 39] which, as discussed below, provide a path integral approach to quantum gravity. Over the last four years, there has been extensive work in this area, discussed in the articles by Rovelli, Speziale, Baratin, Perini, Fairbairn, Bianchi, and Kaminski in this volume. Transition amplitudes from path integrals can be used to restrict the choice of the Hamiltonian constraint operator in the canonical theory. This is a very promising direction and Freidel, Noui, Perez, Rovelli and others have analyzed this issue especially in $2+1$ dimensions. The idea in the fourth approach, due to Varadarajan, Laddha, Henderson, Tomlin and others, is to use insights gained from the analysis of parameterized field theories. Now the emphasis is on drastically reducing the large freedom in the choice of the definition of the Hamiltonian constraint by requiring that the quantum constraint algebra closes, so that one is assured that there is no obstruction to obtaining a large number of simultaneous solutions to all constraints. Because the Poisson bracket between two Hamiltonian constraints is a diffeomorphism constraint, one has to find a viable expression of the operator generating infinitesimal diffeomorphisms. (Until this work, the focus was on the action only of finite diffeomorphisms in the kinematical setup.) Very recently, this program has witnessed promising advances. The Hamiltonian constraint one is led to define shares qualitative features of 'improved dynamics' of loop quantum cosmology that lies at the foundation of the most significant advances in that area.

In this discussion I have focused primarily on pure gravity. In the mid 1990s Brown, Kuchar and Romano had introduced frameworks in which matter fields can be used as 'rods and clocks' thereby providing a natural 'de-parametrization' of the constraints in the classical theory. Giesel, Thiemann, Tambornino, Domagala, Kaminski, Lewandowski, Husain and Pawlowski have used these considerations as the point of departure to construct loop quantum gravity theories for these systems. Deparametrization greatly facilitates the task of finding Dirac observables and makes it easier to interpret the quantum theory. However, as in the Master Constraint program, issues associated with quantization ambiguities still remain and the domain on which matter fields serve as good clocks and rods still needs to be clarified. Further details can be found in the lectures by Giesel and Salhmann.

- Spin foams: Four different avenues to quantum gravity have been used to arrive at spin-foam models (SFMs). The fact that ideas from seemingly unrelated directions converge to the same type of structures and models has provided a strong impetus to the spin foam program. Indeed, currently this is the most active area on the mathematical physics side of loop quantum gravity.

The first avenue is the Hamiltonian approach to loop quantum gravity [38, 39, 45]. By mimicking the procedure that led Feynman [1] to a sum over histories formulation of quantum mechanics, Rovelli and Reisenberger proposed a space-time formulation of this theory. This work launched the spin-foam program. The second route stems from the fact that the starting point in canonical 
loop quantum gravity is a rewriting of classical general relativity that emphasizes connections over metrics [38]. Therefore in the passage to quantum theory it is natural to begin with the path integral formulation of appropriate gauge theories. A particularly natural candidate is the topological B-F theory because in 3 space-time dimensions it is equivalent to Einstein gravity, and in higher dimensions general relativity can be regarded as a constrained BF theory [32, 35]. The well-controlled path integral formulation of the BF theory provided the second avenue and led to the SFM of Barrett and Crane.

The third route comes from the Ponzano-Regge model of 3-dimensional gravity that inspired Regge calculus in higher dimensions. Here one begins with a simplicial decomposition of the space-time manifold, describes its discrete Riemannian geometry using edge lengths and deficit angles and constructs a path integral in terms of them. If one uses holonomies and discrete areas of loop quantum gravity in place of edge lengths, one is again led to a spin foam. These three routes are inspired by various aspects of general relativity. The fourth avenue starts from approaches to quantum gravity in which gravity is to emerge from a more fundamental theory based on abstract structures that, to begin with, have nothing to do with space-time geometry. Examples are matrix models for 2-dimensional gravity and their extension to 3-dimensions - the Boulatov model where the basic object is a field on a group manifold rather than a matrix. The Boulatov model was further generalized to a group field theory tailored to 4-dimensional gravity [39, 48]. The perturbative expansion of this group field theory turned out be very closely related to 'vertex expansions' in SFMs. Thus the SFMs lie at a junction where four apparently distinct paths to quantum gravity meet. Through contributions of many researchers it has now become an active research area (see, e.g., [35, 39]).

Four years ago, two groups, Engle-Livine-Pereira-Rovelli, and Freidel-Krasnov, put forward precise proposals for the sum over quantum geometries that could provide detailed dynamics in loop quantum gravity. The motivations were different but for the physically interesting values of the Babero-Immirzi parameter (selected, e.g., by the black hole entropy considerations), the two proposals agree. This is an improvement over the earlier Barrett-Crane model which cured some of the problems faced by that model. Perhaps more importantly, thanks to the generalizations by Kaminski, Kisielowski and Lewandowski, the canonical and path integral approaches have been brought closer to one another: they use the same kinematics. However, there does not yet exit a systematic 'derivation' leading to this proposal starting from classical general relativity, say, along the lines used in textbooks to arrive at the path integral formulation of gauge theories. Nonetheless the program has attracted a large number of researchers because: i) there do exist semi-heuristic considerations motivating the passage; ii) as I indicated above, it can be arrived at from four different avenues; and iii) Detailed asymptotic analysis by Barrett, Hellmann, Dowdall, Fairbairn, Pereira and others strongly indicates that these models have the correct classical limit; and, iv) Because of the use of quantum geometry - more precisely because there is a non-zero area gapthis sum over quantum geometries has no ultra-violet divergences. More recently, Fairbairn, Meusberger, Han and others have extended these considerations to include a cosmological constant by a natural use of quantum groups. It is then argued that, for a given 2-simplex, the sum is also infra-red finite.

However, the issue of whether to sum over distinct 2-complexes or to take an appropriate 'continuum limit' is still debated and it is not known whether the final result would be finite in either 
case. ${ }^{10}$ In the cosmological mini-superspaces, the situation is well-controlled: under a single assumption that a sum and an integral can be interchanged, the analog of the sum over 2-complexes (called the vertex expansion in the spin foam literature) has been shown to converge, and furthermore, converge to the 'correct' result that is already known from a well-established Hamiltonian theory [54].

A much more detailed discussion of spin foams can be found in the lectures by Rovelli, Speziale, Baratin, Perini, Fairbairn, Bianchi, and Kaminski at this school.

\subsection{Challenges and Opportunities}

Developments summarized so far should suffice to provide a sense of the extent to which advances in loop quantum gravity already provide an avenue to a non-perturbative and background independent formulation of quantum gravity. I will conclude by providing an illustrative list of the open issues. Some of these are currently driving the field while others provide challenges and opportunities for further work. This discussion assumes that the reader is familiar with basic ideas behind current research in loop quantum gravity.

\subsubsection{Foundations}

- Hamiltonian Theory: In section 2.2 I outlined four strategies that are being used to extract quantum dynamics. I will now sketch another avenue, inspired in part by the success of loop quantum cosmology, that has been proposed by Domagala, Giesel, Kaminski and Lewandowski. In loop quantum cosmology, a massless scalar field often serves as an 'internal clock' with respect to which observables of physical interest evolve [54]. The idea is to take over this strategy to full quantum gravity by focusing on general relativity coupled with a massless scalar field. This is a particularly interesting system because, already in the 1990s, Kuchar and Romano showed that one can rearrange the constraints of this system so that they form a true Lie algebra, where the Hamiltonian constraints Poisson commute with each other on the entire phase space. Interestingly, under seemingly mild assumptions one can show that solutions of this system admit space-like foliations on which $\phi$ is constant. Consequently, even though the system has infinitely many degrees of freedom, as in $L Q C$ one can use $\phi$ as a relational time variable. With $\mathbb{T}^{3}$ spatial topology for definiteness, one can decompose all fields into homogeneous and purely inhomogeneous modes. If one were to truncate the system by setting the inhomogeneous modes to zero, the resulting quantum theory would be precisely the loop quantum cosmology of Bianchi I models that has been analyzed in detail by Ashtekar and Wilson-Ewing. One might imagine incorporating the inhomogeneous modes using the 'hybrid' quantization scheme that has been successfully used in the Gowdy models by Mena, Martin-Benito, Pawlowski and others, although it will have to be non-trivially generalized to handle the fact that there are no Killing fields. As in the Gowdy models, this will likely involve some gauge fixing of the diffeomorphism and Gauss constraints. Even if these gauge fixing strategies do not work globally on the full phase space, one should still obtain a quantum theory tailored to a 'non-linear neighborhood' of FLRW or Bianchi I space-times. Finally, effective equations for this system would also provide valuable insights into the singularity resolution (which we expect to

\footnotetext{
${ }^{10}$ Mathematically, this situation is somewhat reminiscent to perturbative super-string theory, where there is evidence that each term in the expansion is finite but the sum is not controlled.
} 
persist in an appropriate, well-defined sense). In particular, one would be able to compare and contrast their prediction with the simple BKL behavior near the general relativistic singularity, found by Andersson and Rendall for this system. More generally, this analysis will enable one to place loop quantum cosmology in the setting of full loop quantum gravity.

A second important open issue is to find restrictions on matter fields and their couplings to gravity for which this non-perturbative quantization can be carried out to a satisfactory conclusion. Supersymmetry, for example, is known to allow only very specific matter content. Recent work by Bodendorfer, Thiemann and Thurn has opened a fresh window for this analysis. A second possibility is suggested by the analysis of the closure of the constraint algebra in quantum theory. When it is extended to allow for matter couplings, the recent work by Varadarajan, Laddha, and Tomlin referred to in section 2.2 could provide a promising approach to explore this issue in detail. Finally, as mentioned in section 1.1, the renormalization group approach has provided interesting hints. Specifically, Reuter et al have presented significant evidence for a non-trivial fixed point for vacuum general relativity in 4 dimensions [47]. When matter sources are included, it continues to exist only when the matter content and couplings are suitably restricted. For scalar fields, in particular, Percacci and Perini have found that polynomial couplings (beyond the quadratic term in the action) are ruled out, an intriguing result that may 'explain' the triviality of such theories in Minkowski space-times [42]. Are there similar constraints coming from loop quantum gravity?

- Spin foams: As discussed in section 2.2, the spin foam program has made significant advances over the last four years. Results on the classical limit and finiteness of the sum over histories for a fixed 2-complex are especially encouraging. Therefore it is now appropriate to invest time and effort on key foundational issues.

First, we need a better understanding of the physical meaning of the 'vertex expansion' that results when one sums over arbitrary 2-complexes. In particular, is there a systematic physical approximation that lets us terminate the sum after a finite number of terms? In group field theory each term is multiplied by a power of the coupling constant $[39,48]$ but the physical meaning of this coupling constant in space-time terms is not known. Analysis by Ashtekar, Campiglia and Henderson in the cosmological context bears out an early suggestion of Oriti that the coupling constant is related to the cosmological constant. In the full theory, Fairbairn, Meusberger, Han and others have shown that the cosmological constant can be incorporated using quantum groups (which also makes the spin foam sum infrared finite for a fixed 2-complex). It is then natural to ask if there is a precise sense in interpreting the vertex expansion as a perturbation series in a parameter physically related to the cosmological constant also in the full theory.

Second, as I mentioned in section 2.2, the issue of whether one should actually sum over various 2-complexes (i.e., add up all terms in the vertex expansion), or take an appropriately defined continuum limit is still open. Rovelli and Smerlak have argued that there is a precise sense in which the two procedures coincide. But so far there is no control over the sum and experts in rigorous field theory have expressed the concern that, unless a new principle is invoked, the number of terms may grow uncontrollably as one increases the number of vertices. Recent work on group field theory by Oriti, Rivasseau, Gurau, Krajewski and others may help streamline this analysis and provide the necessary mathematical control.

Finally, because the EPRL and FK models are motivated from the BF theory, they inherit 
certain ('Plebanski') sectors which classically do not correspond to general relativity. In addition, analysis of cosmological spin foams re-enforces an early idea due to Oriti that one should only sum over 'time oriented' quantum geometries. Some of these issues are now being analyzed in detail by Engle and others. But more work is needed on these basic conceptual issues.

- Low energy physics: In low energy physics one uses quantum field theory on given background space-times. Therefore one is naturally led to ask if this theory can be arrived at by starting from loop quantum gravity and making systematic approximations. Here, a number of interesting challenges appear to be within reach. Fock states have been isolated in the polymer framework [38] and elements of quantum field theory on quantum geometry have been introduced [45]. These developments lead to concrete questions. For example, in quantum field theory in flat space-times, the Hamiltonian and other operators are regularized through normal ordering. For quantum field theory on quantum geometry, on the other hand, the Hamiltonians are expected to be manifestly finite $[45,38]$. Can one then show that, in a suitable approximation, normal ordered operators in the Minkowski continuum arise naturally from these finite operators? Can one 'explain' why the so-called Hadamard states of quantum field theory in curved space-times are special? These considerations could also provide valuable hints for the construction of viable semi-classical states of quantum geometry.

Since quantum field theory in FLRW space-times plays such an important role in the physics of the early universe, it is especially important to know if can be systematically derived from loop quantum gravity. A number of obstacles immediately come to mind. In the standard treatment of quantum fields on cosmological space-times, one typically works with conformal or proper time, makes a heavy use of the causal structure made available by the fixed background space-time, and discusses dynamics as an unitary evolution in the chosen time variable. In quantum geometry state of loop quantum cosmology, none of these structures are available. Even in the 'deparameterized picture' it is a scalar field that plays the role of internal time; proper and conformal times are at best operators. Even when the quantum state is sharply peaked on an effective solution, we have only a probability distribution for various space-time geometries; we do not have a single, welldefined, classical causal structure. Finally, in loop quantum gravity, dynamics is teased out of the constraint while in quantum field theory in curved space-times it is dictated by a Hamiltonian. These obstacles seem formidable at first, Ashtekar, Kaminski and Lewandowski have shown that they can be overcome if one works with spatially compact topology and focuses just on a finite number of modes of the test field. The first assumption frees one from infra-red issues which can be faced later while the second restriction was motivated by the fact that, in the inflationary scenario, only a finite number of modes of perturbations are relevant to observations. It is important to remove these restrictions and use the resulting framework to analyze the questions on the quantum gravity origin of Hadamard states and of the adiabatic regularization procedure routinely used in cosmology.

\subsubsection{Applications}

- The very early universe: Because the initial motivations for inflation are not as strong as they are often portrayed to be, several prominent relativists were put off by the idea. As a consequence, recent developments in the inflationary paradigm have not drawn due attention in general relativity 
circles. In my view, there is a compelling case to take the paradigm seriously: it predicted the main features of inhomogeneities in the cosmic microwave background (CMB) which were subsequently observed and which serve as seeds for structure formation.

Let me first explain this point in some detail. Note first that one analyzes CMB inhomogeneities in terms of their Fourier modes and observationally relevant wave numbers are in a finite range, say $\Delta k$. Using this fact, we can write down the four assumptions on which the inflationary scenario is based:

1) Some time in its very early history, the universe underwent a phase of accelerated expansion during which the Hubble parameter $\mathrm{H}$ was nearly constant.

2) During this phase the universe is well-described by a FLRW background space-time together with linear perturbations.

3) A few e-foldings before the longest wave length mode in the family $\Delta k$ under consideration exited the Hubble radius, these Fourier modes of quantum fields describing perturbations were in the Bunch-Davis vacuum.

4) Soon after a mode exited the Hubble radius, its quantum fluctuation can be regarded as a classical perturbation and evolved via linearized Einstein's equations.

Then QFT on FLRW space-times and classical general relativity imply the existence of tiny inhomogeneities in CMB which have been seen by the 7 year WMAP data. Numerical simulations show that these seeds grow to yield the large scale structure that is observed today. Although the assumptions are by no means compelling, the overall economy of thought it is nonetheless impressive. In particular, in this paradigm, the origin of the large scale structure of the universe lies just in vacuum fluctuations! Therefore, it is of considerable interest to attempt to provide a quantum gravity completion of this paradigm.

The issues that are left open by this standard paradigm are of two types: Particle Physics issues and Quantum Gravity issues. Let me focus on the second for now:

1) Initial singularity: The paradigm assumes classical general relativity and theorems due to Borde, Guth and Vilenkin then imply that space-time had an initial big bang singularity. For reasons discussed in section 1, this is an artifact of using general relativity in domains where it is not applicable. Therefore, one needs a viable treatment of the Planck regime and the corresponding extension of the inflationary paradigm.

2) Probability of inflation: In loop quantum cosmology, the big bang is replaced by a quantum bounce. So it is natural to introduce initial conditions there. Will a generic homogeneous, isotropic initial state for the background, when evolved, encounter a phase of slow roll inflation compatible with the seven year WMAP data?

3) Trans-Planckian issues: In classical general relativity, if we evolve the Fourier modes of interest back in time, they become trans-Planckian. We need a quantum field theory on quantum cosmological space-times to adequately handle them.

4) Observations: The question then is whether the initial quantum state at the bounce, when evolved forward in time agrees sufficiently with the Bunch Davis vacuum at the onset of inflation so as not to contradict the observations. More importantly, are there small deviations which could be observed in future missions?

Recent work by Ashtekar, Sloan, Agullo, Nelson; Barreau, Cailleteau, Grain and Mielczarek; and Fernandez-Mendez, Mena-Marugan,Olmedo and Vehlino, has made notable advances in fac- 
ing these questions [54] but there are ample opportunities for other research that will provide both a viable quantum gravity completion of the inflationary paradigm and potentially observable predictions.

Finally, even if loop quantum gravity does offer a viable quantum gravity completion of the inflationary paradigm, open issues related to particle physics will still remain. In particular: What is the physical origin of the inflaton field? Of the potential one must use to get a sufficiently long slow roll? Is there only one inflaton or many? If many, what are their interaction? What are the couplings that produce the known particles as the inflaton oscillates around the minimum of the potential at the end of inflation (the so-called 'reheating')? Therefore, it would be healthy to look also for alternatives to inflation. Indeed, in the alternatives that have been advocated by Barndenberger and others involve bouncing models and therefore have similarities with the general loop quantum cosmology paradigm. Because the expansion of the universe from the bounce to the surface of last scattering in the post-bounce branch is much smaller than that in the inflationary scenario, at the bounce, modes of direct interest to the CMB observations now have physical frequencies much below the Planck scale. Therefore, the trans-Planckian issue is avoided and quantum field theory in curved space-times should be viable for these modes. This fact, coupled with the absence of singularity, enables one to calculate a transfer matrix relating modes in the pre-bounce epoch to those in the post-bounce epoch. Under suitable assumptions, Brandenberger and others have shown that this relation gives rise to a nearly scale invariant spectrum of scalar and tensor modes in the post-bounce phase. But the underlying premise in these calculations is that perturbations originate in the distant past of the contracting branch where the geometry is nearly flat and quantum fields representing perturbations are taken to be in their vacuum state. This idea that the entire evolution from the distant past in the contracting phase to the bounce is well described by a homogeneous model with small perturbations is not at all realistic. But since the general paradigm has several attractive features, it would be of considerable interest to investigate whether loop quantum cosmology bounces allow similar alternatives to inflation without having to assume that non-linearities can be neglected throughout the pre-bounce phase.

- Black hole evaporation: The issue of the final state: Black hole thermodynamics was initially developed in the context of stationary black holes. Indeed, until relatively recently, there were very few analytical results on dynamical black holes in classical general relativity. This changed with the advent of dynamical horizons which provide the necessary analytical tools to extract physics from numerical simulations of black hole formation and evaporation. It also led to some new insights on the fundamental side. in particular, it was also shown that the first law can be extended to these time-dependent situations and the leading term in the expression of the entropy is again given by $a_{\mathrm{hor}} / 4 \ell_{\mathrm{Pl}}^{2}$ [41]. Hawking radiation will cause the horizon of a large black hole to shrink very slowly, whence it is reasonable to expect that the description of the quantum horizon geometry can be extended from isolated to dynamical horizons in this phase of the evaporation. The natural question then is: Can one describe in detail the black hole evaporation process and shed light on the issue of information loss?

The space-time diagram of the evaporating black hole, conjectured by Hawking, is shown in the left-hand drawing in Fig. 1. It is based on two ingredients: i) Hawking's original calculation of black hole radiance, in the framework of quantum field theory on a fixed background space-time; 

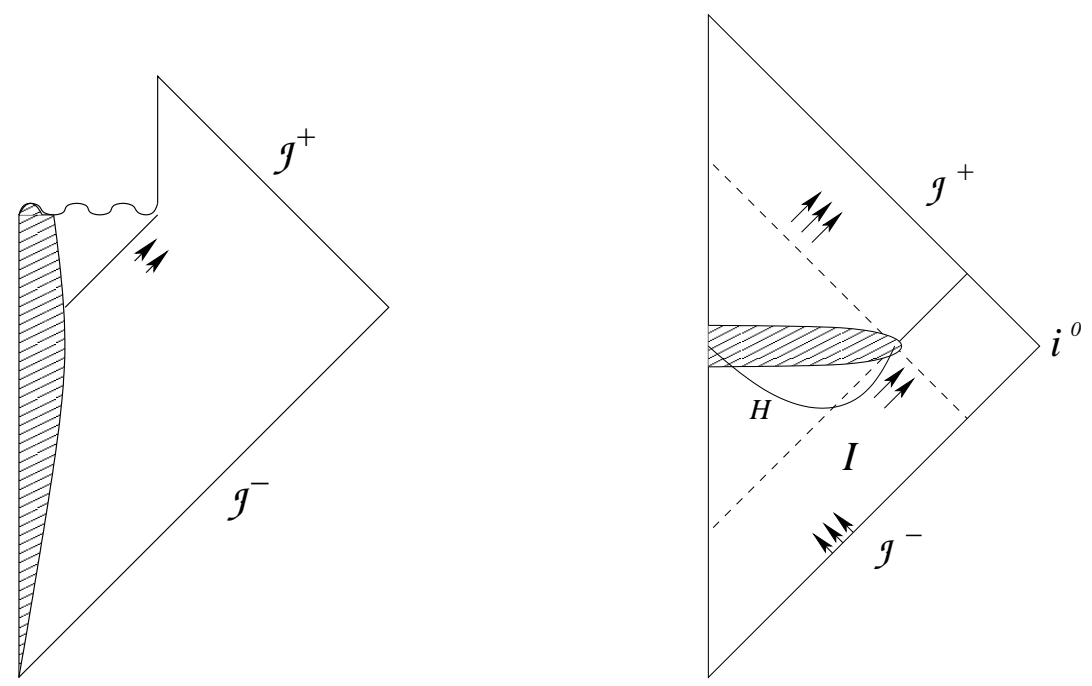

Figure 1: Conjectured space-time diagrams of evaporating black holes in full quantum theory.

(a) Left figure: Standard paradigm, originally proposed by Hawking. Information is lost because part of the incoming state on $\mathscr{I}^{-}$falls into the part of the future singularity that is assumed to persist in the full quantum gravity theory. (b) Right figure: New paradigm motivated by the singularity resolution in LQC. What forms and evaporates is a dynamical horizon $H$. Quantum space-time is larger and the incoming information on the full $\mathscr{I}^{-}$is adequately recovered on the $\mathscr{I}^{-}$of this larger space-time.

and ii) heuristics of back-reaction effects which suggest that the radius of the event horizon must shrink to zero. It is generally argued that the semi-classical process depicted in this figure should be reliable until the very late stages of evaporation when the black hole has shrunk to Planck size and quantum gravity effects become important. Since it takes a very long time for a large black hole to shrink to this size, one then argues that the quantum gravity effects during the last stages of evaporation will not be sufficient to restore the correlations that have been lost due to thermal radiation over such a long period. Thus there is loss of information. Intuitively, the lost information is 'absorbed' by the 'left-over piece' of the final singularity which serves as a new boundary to space-time.

However, loop quantum gravity considerations suggest that this argument is incorrect in two respects. First, the semi-classical picture breaks down not just at the end point of evaporation but in fact all along what is depicted as the final singularity. Using ideas from quantum cosmology, the interior of the Schwarzschild horizon was analyzed in the context of loop quantum gravity by Ashtekar, Bojowald, Modesto, Vandersloot and others. This analysis is not as complete or refined as that in the cosmological context. But the qualitative conclusion that the singularity is resolved due to quantum geometry effects is likely to be robust. If so, the space-time does not have a singularity as its final boundary. The second limitation of this semi-classical picture is its depiction of the event horizon. The notion of an event horizon is teleological and refers to the global structure of space-time. Resolution of the singularity introduces a domain in which there is no classical space-time, whence the notion ceases to be meaningful; it is simply 'transcended' 
in quantum theory. Using these considerations Ashtekar and Bojowald introduced a new paradigm for black hole evaporation in loop quantum gravity, depicted in the right hand drawing of Fig. 1: Now, it is the dynamical horizon that evaporates with emission of quantum radiation, and the initial pure state evolves to a final pure state on the future null infinity of the extended space-time. Thus, there is no information loss. In this paradigm, the semi-classical considerations would not simply dismissed; they would be valid in certain space-time regions and under certain approximations. But for fundamental conceptual issues, they would not be inadequate.

However, this is still only a paradigm and the main challenge is to develop it into a detailed theory. Just as Wheeler's 'It from Bit' ideas were transformed into a detailed theory of quantum horizon geometry, it should be possible to construct a detailed theory of black hole evaporation based on this paradigm. More recently, this paradigm was put on a firm footing by Ashtekar, Taveras and Varadarajan in the case of 2-dimensional black holes first introduced by Callen, Giddings, Harvey and Strominger. The model is interesting especially because its action and equations of motion closely mimic those governing 4-dimensional, spherically symmetric black holes formed by the gravitational collapse of a scalar field. Ashtekar, Pretorius and Ramazanoglu have used a combination of analytical and numerical methods to analyze the mean field approximation in complete detail. It explicitly shows that some of the common assumptions regarding effects of including back reaction, discussed in the last paragraph, are incorrect. This analysis further reinforces the paradigm of the figure on the right. It is therefore of considerable interest to extend all this analysis to four dimensions in the loop quantum gravity setting. The very considerable work on spherically symmetric midi-superspaces by Gambini, Pullin, Bojowald and others will serve as a point of departure for this analysis.

- Contact with low energy physics: Spin foam models provide a convenient arena to discuss issues such as the graviton propagator, $n$ point functions and scattering, that lie at the heart of perturbative treatments. At first, it seems impossible to have non-trivial $n$-point functions in a diffeomorphism invariant theory. Indeed, how could one say that the 2-point function falls off as $1 / r^{n}$ when the distance $r$ between the two points has no diffeomorphism invariant meaning? Thanks to a careful conceptual set-up by Oeckl, Colosi, Rovelli and others, this issue has been satisfactorily resolved. To speak of $n$-point functions, one needs to introduce a boundary state (in which the expectation values are taken) and the notion of distance $r$ descends from the boundary state. Interestingly, a detailed calculation of the 2-point function brought out some limitations of the Barrett-Crane model and provided new impetus for the EPRL and FK models. As Perini's talks at this school showed, these calculations by Bianchi, Ding, Magliaro and Perini strongly indicate that, to the leading order, a graviton propagator with the correct functional form and tensorial structure will arise from these models.

However, these calculations can be improved in a number of respects and their full implications have yet to be properly digested. In particular, one needs a better handle on contributions from 2-complexes with large numbers of vertices and the physics of the sub-leading terms. These terms seem to be sensitive to the choice of the boundary state and there isn't a canonical one representing Minkowski space. Therefore, comparison with the standard perturbation theory in Minkowski space is difficult. This is a fertile and important area for further research. Indeed the key challenge in this area is to 'explain' why perturbative quantum general relativity fails if the theory exists 
non-perturbatively. As mentioned in section 1, heuristically the failure can be traced back to the insistence that the continuum space-time geometry is a good approximation even below the Planck scale. But a more detailed answer is needed. For example, is it because, as developments in the asymptotically safe scenarios indicate [47, 52], the renormalization group has a non-Gaussian fixed point?

- Unification. Finally, there is the issue of unification. At a kinematical level, there is already an unification because the quantum configuration space of general relativity is the same as in gauge theories which govern the strong and electro-weak interactions. But the non-trivial issue is that of dynamics. To conclude, let us consider a speculation. One possibility is to use the 'emergent phenomena' scenario where new degrees of freedom or particles, which were not present in the initial Lagrangian, emerge when one considers excitations of a non-trivial vacuum. For example, one can begin with solids and arrive at phonons; start with superfluids and find rotons; consider superconductors and discover cooper pairs. In loop quantum gravity, the micro-state representing Minkowski space-time will have a highly non-trivial Planck-scale structure. The basic entities will be 1-dimensional and polymer-like. One can argue that, even in absence of a detailed theory, the fluctuations of these 1-dimensional entities should correspond not only to gravitons but also to other particles, including a spin-1 particle, a scalar and an anti-symmetric tensor. These 'emergent states' are likely to play an important role in Minkowskian physics derived from loop quantum gravity. A detailed study of these excitations may well lead to interesting dynamics that includes not only gravity but also a select family of non-gravitational fields. It may also serve as a bridge between loop quantum gravity and string theory. For, string theory has two a priori elements: unexcited strings which carry no quantum numbers and a background space-time. Loop quantum gravity suggests that both could arise from the quantum state of geometry, peaked at Minkowski (or, de Sitter) space. The polymer-like quantum threads which must be woven to create the classical ground state geometries could be interpreted as unexcited strings. Excitations of these strings, in turn, may provide interesting matter couplings for loop quantum gravity.

\subsubsection{Some Final Remarks}

From examples discussed in this section it is clear that loop quantum gravity has witnessed significant advances over the last decade, both in its foundations and applications. It is therefore important for the community to make sustained progress on directions that have already been opened up. Of course one constantly needs an influx of new ideas. But it would be a mistake if a significant fraction of the community focuses on constructing new models every few months, making a first stab and then passing on to the next model. The cumulative results in the main stream development of loop quantum gravity now carry sufficient weight for us to take the basic ideas seriously and continue to develop them by attacking the hard conceptual and technical open issues. Examples of such issues are: Finding principles and strategies to significantly narrow the ambiguities in the definition of the Hamiltonian constraint; extending further explorations of the role of supersymmetry initiated by the Erlangen group; sharpening the set of quantum geometries to sum over, and addressing the problem of convergence in spin foam models; analyzing the renormalization group flows in group field theory; understanding the dependence of the n-point functions on the choice of the boundary state; developing approximation methods to calculate S-matrix from spin foams 
and pin-pointing why the standard perturbative treatments fail; fully incorporating matter fields in spin foams, particularly scalar fields; constructing effective field theories to adequately describe low energy physics; finding the detailed relation between loop quantum gravity and loop quantum cosmology; constructing a detailed completion of the inflationary paradigm in the Planck regime; exploring its observable consequences in the very early universe; ... The list is long enough to keep young researchers busy and happy for quite a while! Furthermore, in this work, it is important to keep focus on physical issues and try to solve problems of direct physical interest. Developing formalism is important because it streamlines the ideas and procedures. But it is not an end in itself. Indeed, it is of little use unless it leads to answers to the long standing physical questions.

Finally, there is a complementary direction. Because the mathematics underlying loop quantum gravity is rigorous, the subject is now begun to make inroads into other areas of mathematical physics and mathematics itself. For example, there is now literature on spin networks, quantum topology and computing, spin foams and non commuting geometry, Holst action and asymptotic safety, loop quantum gravity and topos theory, spectral triples over the space of generalized connections etc. from researchers like Kauffmann, Marcolli, Reuter, Dahlen, Aastrup and Grimstrup from outside the traditional loop quantum gravity community. Conversely, some loop quantum gravity researchers such as Ma, Gambini, Pullin, Singh, Dittrich, Freidel and Fleischhack are applying techniques developed in the field to other areas such as the $f(R)$ theories that rose to prominence in cosmology, generalized quantum mechanics, statistical mechanics, non-commutative geometry, gauge theories and geometry. Thus there is ample evidence that the subject is now sufficiently mature to have applications to other areas. In these explorations, it is important to focus on problems that other communities consider as important in their areas. In my view, this 'outward bound' spirit is the second pillar on which further development of the field will rest.

\section{Acknowledgements:}

My understanding of classical and quantum gravity has deepened through discussions with a large number of colleagues; many of whom were at the Zakopane School. Parts of this overview are updated versions of some of the material in author's article [46]. I would like to thank Miguel Campiglia, Kristina Giesel, Alok Laddha and especially Carlo Rovelli for their comments on the manuscript. This work was supported in part by the European Science Foundation through its network Quantum Geometry and Quantum Gravity, NSF grant PHY 0090091 and the Eberly research funds of The Pennsylvania State University.

\section{References}

[1] R. P. Feynman, Space-time approach to non-relativistic quantum mechanics, Rev. Mod. Phys. 20, 367-387 (1948)

[2] Arnowitt R, Deser S and Misner C W 1962 The dynamics of general relativity, in Gravitation: An introduction to current research ed Witten L (John Wiley, New York)

[3] Wheeler J A 1962 Geometrodynamics, (Academic Press, New York)

[4] Wheeler J A 1964 Geometrodynamics and the issue of the final state Relativity, Groupos and Topology eds DeWitt C M and DeWitt B S (Gordon and Breach, New York) 
[5] Komar A 1970 Quantization program for general relativity, in Relativity Carmeli M, Fickler S. I. and Witten L (eds) (Plenum, New York)

[6] Ashtekar A and Geroch R 1974 Quantum theory of gravitation, Rep. Prog. Phys. 37 1211-1256

[7] Weinberg S 1972 Gravitation and Cosmology (John Wiley, New York)

[8] DeWitt B S 1972 Covariant quantum geometrodynamics, in Magic Without Magic: John Archibald Wheeler ed Klauder J R (W. H. Freeman, San Fransisco)

[9] Isham C. J. 1975 An introduction to quantum gravity, in Quantum Gravity, An Oxford Symposium Isham C J, Penrose R and Sciama D W (Clarendon Press, Oxford)

[10] Duff M 1975 Covariant qauantization in Quantum Gravity, An Oxford Symposium Isham C J, Penrose R and Sciama D W (Clarendon Press, Oxford)

[11] Penrose R 1975 Twistor theory, its aims and achievements Quantum Gravity, An Oxford Symposium Isham C J, Penrose R and Sciama D W (Clarendon Press, Oxford)

[12] Israel W and Hawking S W eds 1980 General Relativity, An Einstein Centenary Survey (Cambridge UP, Cambridge)

[13] Bergmann P G and Komar A 1980 The phase space formulation of general relativity and approaches toward its canonical quantization General Relativity and Gravitation vol 1, On Hundred Years after the Birth of Albert Einstein, Held A ed (Plenum, New York)

[14] Wolf H (ed) 1980 Some Strangeness in Proportion (Addison Wesley, Reading)

[15] Hawking S W 1980 Is End In Sight for Theoretical Physics?: An Inaugural Address (Cambridge UP, Cambridge)

[16] Kuchař K 1981 Canonical methods of quantization, in Quantum Gravity 2, A Second Oxford Symposium Isham C J, Penrose R and Sciama D W (Clarendon Press, Oxford)

[17] Isham C J 1981 Quantum gravity-An overview, in Quantum Gravity 2, A Second Oxford Symposium Isham C J, Penrose R and Sciama D W (Clarendon Press, Oxford)

[18] Ko M, Ludvigsen M, Newman E T and Tod P 1981 The theory of $\mathscr{H}$ space Phys. Rep. 71 51-139

[19] Ashtekar A 1984 Asymptotic quantization (Bibliopolis, Naples); also available at http://cgpg.gravity.psu.edu/research/asymquant-book.pdf

[20] Greene M B, Schwarz J H and Witten E 1987 Superstring theory, volumes 1 and 2 (Cambridge UP, Cambridge)

[21] R. Penrose and W. Rindler, Spinors and space-times, Vol 2, (cambridge University Press, Cambridge 1988)

[22] Ashtekar A 1991 Lectures on non-perturbative canonical gravity, Notes prepared in collaboration with R. S. Tate (World Scientific, Singapore)

[23] Ashtekar A Mathematical problems of non-perturbative quantum general relativity in Gravitation and Quantizations: Proceedings of the 1992 Les Houches summer school eds Julia B and Zinn-Justin J (Elsevier, Amsterdam); also available as gr-qc/9302024

[24] Williams R W and Tucker P M 1992 Regge calculus: a brief review and bibliography, Class. Quant. Grav. 9 1409-1422

[25] Connes A 1994 Non-commutative geometry, (Academic Press, New York) 
[26] Baez J and Muniain J P 1994 Gauge fields, knots and gravity (World Scientific, Singapore)

[27] Wald R M 1994 Quantum field theory in curved space-time and black hole thermodynamics (Chicago UP, Chicago)

[28] Gambini R and Pullin J 1996 Loops, knots, gauge theories and quantum gravity (Cambridge UP, Cambridge)

[29] Carlip S 1998 Quantum gravity in 2+1 dimensions (Cambrige UP, Cambridge)

[30] Loll R 1998 Discrete approaches to quantum gravity in four dimensions Living Rev. Rel. 113

[31] Polchinski, J 1998 String Theory, volumes 1 and 2, (Cambridge UP, Cambridge)

[32] Baez J C 1999 An introduction to spin foam models of quantum gravity and BF theory, Lect. Notes Phys. 543 (2000) 25-94, arXiv: gr-qc/ 9905087

[33] Ashtekar A 2000 Quantum mechanics of geometry, in The Universe: Visions and Perspectives, eds Dadhich N and Kembhavi A (Kluwer Academic, Dordretch); gr-qc/9901023

[34] Wald R M 2001 Black hole thermodynamics, Living Rev. Rel. 6

[35] Perez A 2003 Spin foam models for quantum gravity Class. Quant. Grav. 20 R43-R104

[36] Klauder J 2003 Affine quantum gravity Int. J. Mod. Phys. D12 1769-1774

[37] Sorkin R 2003 Causal sets: discrete gravity, gr-qc/0309009

[38] Ashtekar A and Lewandowski L 2004 Background independent quantum gravity: A status report, Class. Quant. Grav. 21 R53-R152

[39] Rovelli C 2004 Quantum Gravity (Cambridge University Press, Cambridge)

[40] Ambjorn J, Jurkiewicz J and Loll R 2004 Emergence of a 4D world from causal quantum gravity,

[41] Ashtekar A and Krishnan B 2004 Isolated and Dynamical horizons and their properties, Living Rev. Rel.7:10

[42] Perini D 2004 The asymptotic safety scenario forgravity and matter Ph.D. Dissertation, SISSA

[43] Penrose R 2005 The twistor approach to space-time structure, in: 100 Years of Relativity, ed Ashtekar A (World Scientific, Singapore)

[44] Dowker F 2005 Causal sets and the deep structure of space-time, in: 100 Years of Relativity, ed Ashtekar A (World Scientific, Singapore)

[45] Thiemann T 2005 Introduction to modern canonical quantum general relativity (Cambridge University Press, Cambridge) Phys.Rev.Lett. 93, 131301

[46] Ashtekar 2005 A gravity and the Quantum, New J Phys, 198-232

[47] Niedermaier M and Reuter M 2006 The asymptotic safety scenario in quantum gravity, Liv. Rev. 9

[48] D. Oriti, The group field theory approach to quantum gravity, arXiv:gr-qc/0607032

[49] Horowitz G T and Polchinski J 2009 Gauge theory/grvity duality, in Approaches to Quantum Gravity, ed oriti D (Cambridge UP, Cambridge); also available at arXiv: gr-qc/0602037v3

[50] Gambini R and Pullin J 2005 Consistent discretizations as a road to quantum gravity, in Approaches to Quantum Gravity, ed oriti D (Cambridge UP, Cambridge); also available at arXiv:gr-qc/0512065 
[51] Isham C J 2010 Topos methods in the foundations of physics, arXiv: 1004.3564

[52] Daum J M and Reuter M 2010 Renormalization group flow of the Holst Action arXiv:1012.4280;

Running Immirzi parameter and asymptotic safety, arXiv:1111.1000

[53] Gambini R and Pullin J 2011 A First Course in Loop Quantum Gravity, (Oxford University press, Oxford)

[54] Ashtekar A and Singh P 2011 Loop quantum cosmology: A status report, Class. Quant. Grav. 28 213001

[55] Bern Z, Carrasco J, Dixon L, Johansson H and Roiban, R., Amplitudes and ultraviolet behavior in $\mathscr{N}=8$ supergravity, arXiv: 1103.1848

[56] Dahlen T 2011 A Topos model for loop quantum gravity, arXiv:1111. 5685 